\title{
Square wave jerks in Parkinsonian syndromes
}

\author{
O Rascol, U Sabatini, M Simonetta-Moreau, J L Montastruc, A Rascol, M Clanet
}

\begin{abstract}
The frequency of square wave jerks (SWJ) was compared in eight patients with progressive supranuclear palsy (PSP), 25 patients with multiple system atrophy or Parkinson's disease plus (MSA/PP), 85 patients with idiopathic Parkinson's disease (PD) and 20 agematched normal volunteers. In the control group, the mean (SD) SWJ frequency (SWJ larger than $1^{\circ}$ amplitude) was $2 \cdot 3(2 \cdot 4) /$ min. Abnormal ocular fixation (SWJ frequency $>10 / \mathrm{min}$ ) was observed in a large proportion of PSP patients $(7 / 8)$ and of MSA/PP patients $(16 / 25)$ but in few $P D$ patients $(13 / 85)$. In the group of PD patients with abnormal ocular fixation, freezing of gait, falls and instability were more severe than in the group of PD patients with normal fixation. The study of ocular fixation may help to differentiate PD clinically from other Parkinsonian syndromes. SWJ are probably not related to the central degeneration of the dopaminergic nigrostriatal pathway observed in PD.
\end{abstract}

Square wave jerks (SWJ) are defined as saccadic intrusions observed during fixation. They correspond to sporadic involuntary horizontal conjugate saccades away from the intended position of fixation, followed after an interval by a saccadic return to the fixation position. ${ }^{1} \mathrm{SWJ}$ occurring in darkness without fixation are physiological. Many normal subjects have low amplitude, low frequency SWJ. ${ }^{2}$ Elderly subjects have more frequent SWJ. ${ }^{2}$ Pathological SWJ have been reported as a common feature of cerebellar system diseases, ${ }^{3}$ progressive supranuclear palsy (PSP), ${ }^{4-6}$ Huntington's chorea, ${ }^{78}$ Alzheimer's disease, ${ }^{910}$ focal cerebral lesions ${ }^{3}$ and non paralytic strabismus. ${ }^{11}$ In Parkinson's disease (PD), several abnormalities of ocular saccades and smooth pursuit movements have been described $^{1213}$ but very few data are available on SWJ. To our knowledge, only one study ${ }^{14}$ reported that $S W J$ could be considered a feature of the disease. We studied SWJ in a population of patients with different "degenerative" "Parkinsonian" disorders.

Service de Neurologie A Rascol

Correspondence to: Dr Rascol, Laboratoire de Pharmacologie Médicale et clinique, Inserm U317, Faculté de Médecine, 37 allées Jules-Guesde 31073 , Toulouse Cedex, France Received 15 September 1989 and in final revised form 19 October 1990

Accepted 7 November 1990

\section{Patients and methods}

One hundred and eighteen patients and 20 age . Patients were included if they pystem presenting extrapyramid symptoms. Patients with severe dementia, drug-induced or post encephalitic Parkinsonism, patients who had undergone neurosurgery, those with neurological disease other than "Parkinsonism" and patients unable to collaborate in the oculomotor study (because of poor vision, too much severe oculomotor abnormalities, poor cooperation) were excluded. Hypnotic and anxiolytic drugs were prescribed during the two days preceding the examination. All anti-Parkinsonian medication was withheld for at least 12 hours before evaluation to observe when the patients were "off'. Patients were classified in three different groups according to clinical diagnostic criteria.

The first group included eight patients with probable progressive supranuclear palsy (PSP). These patients presented axial rigidity without tremor, poor response to levodopa or subcutaneous (SC) apomorphine, slow vertical upward and downward saccades, unsteady gait with falls and moderate intellectual impairment.

The second group included 25 patients with the clinical diagnosis of probable multiple system atrophy (MSA) or Parkinson's plus (PP). These patients corresponded to an heterogeneous group of patients with "atypical" Parkinsonian syndromes previously defined as Shy Drager disease, olivopontocerebellar atrophy, or corticobasal degeneration. All the patients included in this group were characterised by the presence of an extrapyramidal syndrome associated with one or more of the following criteria: cerebellar or pyramidal symptoms of unidentified cause, severe autonomic failure, negative response to large doses of levodopa or SC apomorphine, isolated "ataxic-Parkinsonian" or "freezing" gait.

The third group corresponded to 85 patients with the clinical diagnosis of probable "idiopathic" or "Lewy body" Parkinson's disease; such patients were included if they did not present any of the preceding criteria for the diagnosis of PSP and MSA/PP, if they presented at least two of the three cardinal syptoms of Parkinsonism (rest tremor, limb akinesia or limb rigidity) and a clear improvement induced by levodopa or SC apomorphine. The motor status of these PD patients was evaluated according to the Hoehn and Yahr stages ${ }^{15}$ and the Unified Parkinson's disease rating scale (UPDRS, version 1 approximately to version 3 October 1984). ${ }^{16}$ The UPDRS permitted a quantification of the global score of the motor examination (items 8 to 31 ), the scores of the three main dopa-responsive symptoms of the disease, that is, rest tremor (item 20), akinesia (sum of items 19, 23-27, 31) and rigidity (item 22) and the scores of other symptoms such as 


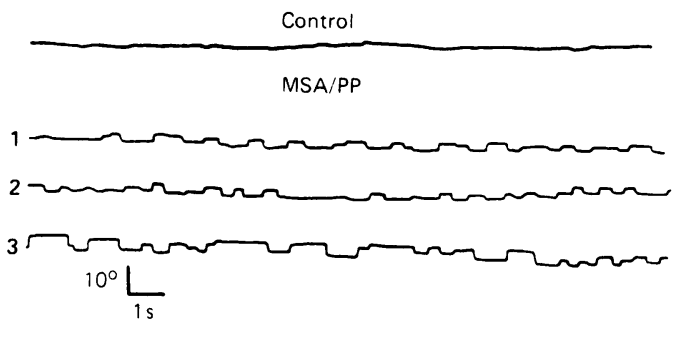

Figure 1 Examples of electro-ocular recordings during fixation of an illuminated target in darkness in one control subject and three MSA/PP patients. No saccadic intrusion larger than $1^{\circ}$ amplitude was observed in the control subject (a 65 year old man). On the contrary, frequent $S W J>1^{\circ}$ were recorded in the three different examples of $M S A / P P$ patients $(1=$ a 63 year old male with the clinical diagnosis of Shy Drager disease, that is levodopa responsive Parkinsonism + severe orthostatic hypotension; 2 = a 66 year old male with a pure freezing of the gait unresponsive to levodopa and $S C$

apomorphine; $3=a 73$ year old male with a levodopa responsive Parkinsonism combined with mild dementia and bilateral Babinski sign).

freezing (item 14), falling (item 13), speech (item 18), posture (item 28) and postural stability (item 30 ).

SWJ were studied using a Pathfinder II (Nicolet Biomedical Instruments with the Nicolet Pathfinder Automated Electronystagmography package). Electro-oculography (EOG) was used for detecting eye movements using silver/silver chloride electrodes, placed near the eyes, which recorded the summed horizontal movements of both eyes and the vertical movements of the left eye. ${ }^{11}$ The heads of the subjects were restrained in mid position. Calibration ( $10^{\circ}$ excentration) and EOG data were registered in total darkness. For fixation and SWJ studies, patients were constantly encouraged during the test $(60 \mathrm{~s})$ to look at a central mid position illuminated target (light emitting diode, Nicolet LT 100 lightbar stimulator). The Pathfinder II provided linear recording over a range \pm 20 degrees. EOG amplifiers were $\mathrm{AC}$ with a long time base (time constant $=15.9 \mathrm{~s})$. Bandwidths were 0.01-40 $\mathrm{Hz}$. Sampling rate of the computer was $100 \mathrm{~Hz}$. Only horizontal SWJ were analysed. SWJ smaller than $1^{\circ}$ amplitude were not considered because of the limits of the amplitude resolution of the apparatus. The frequency of SWJ larger than $1^{\circ}$ was calculated.

The Chi square test and the non-parametric Mann and Whitney U test were used. Results are expressed as mean values (SD).

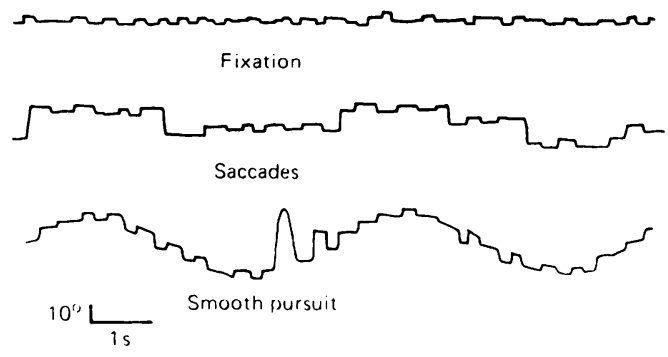

Figure 2 Examples of $S W J$ recorded during fixation and superimposed to saccadic and smooth pursuit ocular movements in a 45 year old female with the clinical diagnosis of olivopontocerebellar atrophy, that is, static and kinetic cerebellar syndrome combined with rest tremor and bilateral pyramidal syndrome.

\section{Results}

No age difference was observed between control subjects [60 (5) years], PSP patients [63 (7) years], MSA/PP patients [62 (14) years] and PD patients [61 (7) years].

In the control group, SWJ were observed with a mean frequency of $2.3(2.4) \mathrm{SWJ} / \mathrm{min}$ ute. Patients with more than 10 SWJ per minute (that is, mean SWJ frequency of the control group $+3 \mathrm{SD}$ ) were considered as patients with ocular fixation impairment (SWJ + patients). Conversely, patients with less than $10 \mathrm{SWJ} /$ minute were considered to have no significant saccadic intrusions (SWJ patients). According to these definitions, seven out of the eight PSP patients, 16 out of the 25 MSA/PP patients and 13 out of the 85 PD patients were SWJ + patients. SWJ + patients were observed with significantly greater frequency in the PSP and MSA/PP groups than in the PD group $(p<0.001)$. In SWJ + patients, SWJ occurred with a mean frequency of $54(15) /$ minute in the PSP group, 42 (13)/minute in the MSA/PP group and 45 (11)/minute in the PD group. There was no difference between the three groups. Most SWJ + patients had a SWJ frequency greater than $30 /$ minutes and it was easy to differentiate SWJ + and SWJ - patients.

Within the MSA/PP and the PD populations SWJ + and SWJ - patients were compared. In the MSA/PP group, no difference was observed between SWJ + and SWJ - patients according to the mean age [62 (15) years versus 61 (14) years], disease duration [10 (8) years versus 6 (3) years], levodopa dose [468 (399) $\mathrm{mg} / \mathrm{d}$ versus $650(241) \mathrm{mg} / \mathrm{d}$ ] or duration of dopa treatment [7 (7) years versus 6 (3) years]. Similarly, no clinical feature could differentiate SWJ + and SWJ - MSA/PP patients except a cerebellar syndrome which was observed in six out of the $16 \mathrm{SWJ}+\mathrm{MSA} / \mathrm{PP}$ patients and in none of the nine SWJ - patients; otherwise pyramidal signs, urinary dysfunction, orthostatic hypotension and/or poor efficacy of

Table Comparison of different parameters and features of the disease in $S W J+$ and $S W J-P D$ patients. No difference was observed in mean age, $P D$ duration, levodopa dose or duration, Hoehn and Yahr stages and total motor examination score of the Unified Parkinson's

Disease Rating Scale (UPDRS). There was also no difference between the three cardinal symptoms best improved by levodopa (akinesia, rigidity, rest tremor). Conversely, the score of three out of five symptoms known to be poorly improved by levodopa (freezing when walking, falls and stability) were significantly more severe in $S W J+$ patients.

\begin{tabular}{|c|c|c|c|}
\hline \multirow[b]{2}{*}{$\begin{array}{l}\text { Age (yrs) } \\
\text { PD duration (yrs) } \\
\text { Dopa duration }(\mathrm{yrs}) \\
\text { Dopa dose (mg/d) } \\
\text { Hoehn and Yahr }\end{array}$} & \multicolumn{3}{|c|}{$S W J+(n=13) S W J-(n=72) p$} \\
\hline & $\begin{array}{c}64(5) \\
7(6) \\
6(7) \\
390(350) \\
2 \cdot 5(1 \cdot 4)\end{array}$ & $\begin{array}{c}61(8) \\
7(6) \\
6(6) \\
420(390) \\
2 \cdot 4(1 \cdot 3)\end{array}$ & $\begin{array}{l}\text { NS } \\
\text { NS } \\
\text { NS } \\
\text { NS } \\
\text { NS }\end{array}$ \\
\hline \multicolumn{4}{|l|}{ UPDRS } \\
\hline $\begin{array}{l}\text { Total score } \\
\text { Akinesia } \\
\text { Rigidity } \\
\text { Tremor } \\
\text { Freezing } \\
\text { Falls } \\
\text { Speech } \\
\text { Posture } \\
\text { Stability }\end{array}$ & $\begin{array}{l}20(9) \\
11(6) \\
1.9(1) \\
2.1(1.4) \\
1.0(1.0) \\
0.8(0.9) \\
1.1(1.1) \\
1.3(1.0) \\
1 \cdot 1(1.0)\end{array}$ & $\begin{array}{l}18(11) \\
10(7) \\
1.8(1) \\
1.8(1.3) \\
0.2(0.8) \\
0.2(0.6) \\
0.9(1.0) \\
1.0(0.9) \\
0.5(0.9)\end{array}$ & $\begin{array}{l}\text { NS } \\
\text { NS } \\
\text { NS } \\
\text { NS } \\
<0 \cdot 01 \\
<0.05 \\
\text { NS } \\
\text { NS } \\
<0.05\end{array}$ \\
\hline
\end{tabular}


dopaminergic drugs were equally observed in both SWJ + and SWJ - MSA/PP patients. Although no quantitative evaluation of cognitive functions was performed, SWJ + patients did not clinically appear to be more or less demented than SWJ - patients.

SWJ + and SWJ - PD patients were also compared (table 1). No difference was observed between mean age, duration or severity of PD, duration or dosage of levodopa. Among the different symptoms of the Parkinsonian syndrome, no significant difference was observed for the three symptoms which are best improved by levodopa (tremor, akinesia, rigidity). On the contrary, freezing when walking, falls and instability were significantly more impaired in SWJ + than in SWJ - PD patients $(\mathrm{p}<0.001, \mathrm{p}<0.05$ and $\mathrm{p}<0.05$ respectively).

\section{Discussion}

According to our data, SWJ frequency was low in the control population. In a previous report, Herisharu and Sharpe observed that SWJ frequency increased with age and was equal to $27(9 \cdot 8) / \mathrm{min}$ in an elderly group of 12 subjects. $^{2}$ This frequency is considerably higher than we have found. This difference is explained by the different experimental conditions of the two studies (electrooculographic amplitude resolution). In this study, only rather large SWJ were considered and saccades of $1^{\circ}$ amplitude or smaller were not quantified. In Herisharu's study, smaller saccades were also considered (mean SWJ amplitude $=1 \cdot 1^{\circ}$ ). Moreover, the control population of the present study was younger (mean age $=60$ years) than that of Herisharu and Sharpe (mean age $=71$ years). In extrapyramidal disorders, SWJ and saccadic intrusions have not been extensively studied except in PSP and Huntington's disease. ${ }^{1}$ SWJ have been repeatedly reported in a number of studies of ocular motor defects in PSP. ${ }^{4-6}$ SWJ occurred in these studies with a frequency of about $1 \mathrm{~Hz}$. This value is comparable to those observed in this study. This suggests that, although the amplitude resolution of our experimental conditions was probably not sensitive enough to quantify the small physiological SWJ occurring in the control group, our technique was able to detect and quantify most of the larger SWJ which are observed in diseases such as PSP. Few published data are available on MSA and PD. Typical SWJ have been described in one case of multisystem degeneration. ${ }^{17}$ White et $a l^{14}$ reported that in 14 patients with PD, SWJ occurred far more frequently than in normal subjects. In this study, however, there was no control for the increased frequency of SWJ in the elderly and therefore SWJ frequency was possibly overestimated.

We observed that SWJ larger than $1^{\circ}$ amplitude were frequently observed in PSP and MSA/PP but were not a common feature of PD. This difference may have some clinical value because $P S P, M S A / P P$ and $P D$ are sometimes difficult to differentiate, specially during the first years of development. ${ }^{18}$ The necessity of premortem pathognomonic diagnostic criteria is crucial because clinical prog- nosis and drug efficacy differ with each disease. Poor response to levodopa or SC apomorphine, pyramidal, cerebellar or dysautonomic symptoms, vertical ocular palsy, posterior fossa atrophy on CT scan have been proposed as exclusion criteria for the diagnosis of PD. ${ }^{19-21}$ None of these criteria are pathognomonic alone but, taken together, their association permits better diagnosis. In this study, using such clinical criteria, nearly $90 \%$ of the PSP and $64 \%$ of the MSA/PP presented abnormal SWJ while $85 \%$ of the PD did not. In this population, no pathological proof of the diagnosis was available. Moreover, several MSA/PP patients $(36 \%)$ did not have SWJ and some PD patients $(15 \%)$ had SWJ. A clinical follow up and a pathological analysis of these cases would be useful. SWJ did not accurately differentiate all the patients and cannot be considered as pathognomonic either. The present data, however, suggest that, taken into account with other criteria, ocular fixation studies could be of clinical value in differentiating PD from other Parkinsonian syndromes.

Physiopathological mechanisms of SWJ and ocular fixation are poorly known. It has been hypothesised that saccadic intrusions could result from abnormal supranuclear trigger signals interrupting omnipause cell activity; this would lead to a release of saccadic burst units of the paramedian pontine reticular formation. ${ }^{22}$ These supranuclear signals could come from various brain areas: SWJ have been observed in different pathological conditions suggesting that the integrity of anterior and posterior cerebral hemispheres, ${ }^{3}$ cerebellum ${ }^{3}$ and basal ganglia ${ }^{78}$ is required for normal ocular fixation. Within the basal ganglia, different extrapyramidal pathways could be differently involved in ocular fixation. For example, SWJ in patients with Huntington's chorea have been attributed to an abnormal control of the caudate nucleus-substantia nigra pars reticulata on saccade "gating" mechanisms, through projections to the paramedian pontine reticular formation via the superior colliculus. ${ }^{13}$ SWJ, in this case, appear to be due to an insufficient tonic inhibition of the nigrocollicular connection..$^{23}$ Conversely, the paucity of SWJ that we observed in PD suggests that the nigrostriatal pathway does not play a crucial role in the genesis of "spontaneous" saccades. The observation that SWJ are a common feature of PSP and MSA/PP is unfortunately less informative because the pathological processes in these diseases are too widespread to allow anatomical or pathophysiological conclusions. We observed that all the MSA/PP patients with cerebellar symptoms had SWJ; this agrees with the theory that cerebellar lesions are frequently associated with abnormal ocular fixation. ${ }^{3}$ Cognitive functions have not been quantitatively assessed in this study; severely demented patients were excluded but it is likely that some patients presented mild or moderate cognitive impairments. SWJ have been described in Alzheimer's disease like dementia $^{910}$ and in hemispheric lesions. ${ }^{3}$ Moreover, the high incidence of errors in an anti-saccade task and the correlation between latency and the frontal dysfunction evaluated by neuropsychological tests shown by Pierrot- 
Deseilligny et $a l^{24}$ suggest that short latencies could result from an impairment of the frontal inhibitory system involved in saccade initiation. A "cortical" mechanism cannot be excluded in some of our patients. Finally, two patients with a levodopa sensitive Parkinsonian syndrome associated with pure severe orthostatic hypotension exhibited typical SWJ. Could lesions of the brainstem alone induce abnormal ocular fixation?

Contrasting with other ocular movements, ${ }^{11}$ it is possible to suggest that ocular fixation is not under dopaminergic control. As already discussed, SWJ are mainly observed in extrapyramidal diseases poorly improved by levodopa while they are less frequent in PD patients well improved by dopaminergic treatments. Moreover, within the PD patients, we observed that SWJ + patients had greater impairment of freezing, falls and instability than SWJ patients. Several authors consider that these "axial" symptoms reflect the degeneration of non-dopaminergic pathways. ${ }^{25-27}$ Conversely, in the same patients, the scores of tremor, rigidity and akinesia (considered to reflect the central dopaminergic deficiency)were not different; this suggests that SWJ + and SWJ - PD patients had similar dopaminergic lesions but that the former had more severe non-dopaminergic lesions.

Finally, the direct study of the effects of dopaminergic drugs on ocular fixation is obviously the best way to address the question. To our knowledge, the occurrence of SWJ after neuroleptic treatment has not been reported. Beside their antidopaminergic properties, these drugs frequently antagonise other receptors such as alpha-adrenergic or muscarinic receptors. Moreover, neuroleptics are used in psychiatric patients who have poorly understood abnormal ocular movements and ocular fixation deficits. ${ }^{28}$ For these reasons, the effects of neuroleptics on ocular movements are difficult to assess. The study of the effects of dopaminergic treatments may be more informative. Although we did not perform this study in all our patients, we could study ocular fixation with and without levodopa treatment in three SWJ patients. While the motor status of these patients was clearly improved, indicating a central effect of the drug, no change in SWJ was observed. Saccadic intrusions or fixation instability have also been reported in one MPTPtreated monkey ${ }^{29}$ but not in two others ${ }^{30}$ and in one out of three humans with MPTP-induced Parkinsonism. ${ }^{31}$ In this last case, no consistent change in the frequency of SWJ was noted after dopamine replacement. Fukazawa et $a l,{ }^{17}$ studied the pharmacological responses of SWJ in a patient with multisystem degeneration. They observed that haloperidol had no effect on SWJ while oscillations almost completely disappeared after administration of diazepam, clonazepam, thiamylal or phenobarbital. This last result agrees with the hypothesis that GABAergic systems may be important in the pathogenesis of saccadic intrusions. However, other systems may also be important since catecholamine depletion induced irrepressible saccadic eye movements in control subjects ${ }^{32}$ and 5 hydroxytryptophan or serotonin can modify the activity of the pontine omnipause neurons in animals. ${ }^{33} 34$
We thank Mr L Darolles for technical assistance, the nurses of the Movement Disorder Unit for taking care of the patients and Mrs P Bontemps for typing the manuscript.

1 Sharpe J, Fletcher W. Saccadic intrusions and oscillations. Can J Neurol Sci 1984;11:426-33.

2 Herishanu Y, Sharpe J. Normal square wave jerks. Invest Ophtalmol Vis Sci 1981;20:268-72.

3 Sharpe J, Herishanu Y, White $O$. Cerebral square wave jerks. Neurology 1982;32:57-62.

4 Troost B, Daroff $R$. The ocular motor defects in progressive supranuclear palsy. Ann Neurol 1977;2:397-403.

5 Chu F, Rheingold D, Cogan D, Williams A. The eye movement disorders of progressive supranuclear palsy. Ophtalmology 1979;86:422-8.

6 Fisk J, Goodale M, Burkhart G, Barnett H. Progressive supranuclear palsy: the relationship between ocular motor dysfunction and psychological test performance. Neurology 1982;32:698-705.

7 Avanzini G, Girotti F, Caraceni T, Spreafico R. Oculomotor disorders in Huntington's chorea. J Neurol Neurosurg Psychiatry 1979;42:581-9.

8 Bollen E, Reulen J, Dentteyer J, Van der Kamp W, Roos R, Buruma $O$. Horizontal and vertical saccadic eye movement abnormalities in Huntington's chorea. JNeurol Sci 1986;74:11-22.

9 Jones A, Friedland R, Koss B, Stark L, Thompkins-Ober B. Saccadic intrusions in Alzheimer-type dementia. J Neurol 1983;229:189-94.

10 Fletcher W, Sharpe J. Smooth pursuit dysfunction in Alzheimer's disease. Neurology 1988;38:272-7.

11 Ciuffreda K, Kneyon R, Stark L. Fixational eye movements in ambliopia and strabismus. J Amer Oto Assoc 1979;50:1251-8.

2 Rascol O, Clanet M, Montastruc JL, Simonetta M, SoulierEsteve MJ, Doyon B, Rascol A. Abnormal ocular movements in Parkinson's disease: evidence for involvement of dopaminergic systems. Brain 1989;112:1193-214.

13 Kennard C, Lueck CJ. Oculomotor abnormalities in diseases of the basal ganglia. Rev Neurol 1989;145:587-95.

14 White O, Saint-Cyr J, Tomlinson R, Sharpe J. Ocular motor deficits in Parkinson's disease: II, Control of the saccadic and smooth pursuit systems. Brain 1983;106:571-87.

15 Hoehn M, Yahr M. Parkinsonism: onset, progression and mortality. Neurology 1967;17:427-42.

16 Fahn S, Elton R and the members of the UPDRS development Committee. Unified Parkinson's disease rating scale. In: Fahn S, Marsden D, Calne D, eds. Recent developments in Parkinson's disease. McMillan Health Information, 1987:153-63.

17 Fukazawa T, Tashiro K, Hamada T, Kase M. Multisystem degeneration: drugs and square wave jerks. Neurology 1986;36:1230-3.

18 Quinn N. Multiple system atrophy-the nature of the beast J Neurol Neurosurg Psychiatry 1989;(suppl.):78-89.

19 Gibb W, Lees A. The significance of the Lewy body in the diagnosis of idiopathic Parkinson's disease. Neuropathol Appl Neurobiol 1989;15:27-44.

$20 \mathrm{Gibb}$ W, Lees A. The relevance of the Lewy body to the pathogenesis of idopathic Parkinson's disease. J Neurol pathogenesis of idopathic Parkinson's
Neurosurg Psychiatry 1988;51:745-52.

21 Hughes A, Lees A, Stern G. Apomorphine test to predict dopaminergic responsiveness in Parkinsonian syndromes. Lancet 1990;ii:32-34.

22 Zee D, Robinson D. A hypothetical explanation of saccadic oscillations. Ann Neurol 1979;5:404-14.

23 Hikosaka $\mathrm{O}$, Wurtz $\mathrm{R}$. The basal ganglia. In: Wurtz, Goldberg, eds. The neurobiology of saccadic eye movements. Amsterdam: Elsevier, 1989:257-81.

24 Pierrot-Deseilligny C, Rivaud S, Pillon B, Fournier E, Agid $Y$. Lateral visually-guided saccades in progressive supranuclear palsy. Brain 1989;112:471-87.

25 Klawans $\mathrm{H}$. Individual manifestations of Parkinson's disease after ten or more years of levodopa. Mov Disorders 1986;1:187-92.

26 Bonnet Am, Loria Y, Saint-Hilaire MH, Lhermitte F, Agid $Y$. Does long-term aggravation of Parkinson's disease result from non dopaminergic lesions? Neurology 1987;37:1539-42.

27 Koller W, Glatt S, Vetere-Overfield B, Hassanein R. Falls and Parkinson's disease. Clin Neuropharmacol 1989;12:98-105.

28 Schmid-Burgk W, Becker W, Jürgens R, Kornhuber $\mathbf{H}$. Saccadic eye movements in psychiatric patients. Neuropsychol 1983;10:193-8.

29 Brooks BA, Fuchs AF, Finocchio D. Saccadic eye movements deficits in the MPTP model of Parkinson's disease. Brain Res 1986;383:402-7.

30 Schultz W, Romo R, Scarnati E, Sundström E, Jonsson G, Studer A. Saccadic reaction times, eye-arm coordination and spontaneous eye movements in normal and MPTPtreated monkeys. Exp Brain Res 1989;78:253-67.

31 Hotson J, Langston E, Langston J. Saccade response to dopamine in human MPTP-induced Parkinsonism. Ann Neurol 1986;20:456-63.

32 Tychsen L, Sitaram N. Catecholamine depletion produces irrepressible saccadic eye movements in normal humans. irrepressible saccadic eye mov
Ann Neurol 1989;25:444-9.

33 Baloh R, Markham C, Furuya N. Inhibition of pontine omnipause neurons in the cat by 5-Hydroxytryptophan Exp Neurol 1982;76:586-93.

34 Furuya N, Ashikawa $H$, Yabe T, Suzuki J. The effects of serotonin on the pontine pause neurons in the cat. $A d v$ Oto-Rhinolaryngol 1988;44:224-8. 